\title{
Fractional Calculus for Solving generalized Abel's Integral Equations using Chebyshev Polynomials
}

\section{H. Saleh \\ Department of \\ Mathematics}

Faculty of Science,

University of Zagazig
S. M. Amer

Department of

Mathematics,

Faculty of Science,

University of Zagazig

\begin{abstract}
In this paper we investigate the numerical solution of Abel's integral equations of the first and second kind by chebychev polynomials of the first, second, third and fourth kinds. Some numerical examples are presented to illustrate the method.
\end{abstract}

\section{General Terms}

Numerical solutions, Fractional integral equations

\section{Keywords}

Singular Volterra integral equation, Abel's integral equation, Fractional calculus, Chebyshev polynomial, Collocation pionts

\section{INTRODUCTION}

Abel's integral equations provide an important tool for modeling a numerous phenomena in basic and engineering sciences such as physics, chemistry, biology, electronics and mechanics $[4,14,20]$. Abel's integral equations often appears in two forms the first and second kind as follows.

$$
f(x)=\int_{0}^{x} \frac{u(t)}{\sqrt{(x-t)}} d t
$$

And

$$
u(t)=f(x)+\int_{0}^{x} \frac{u(t)}{\sqrt{(x-t)}} d t
$$

where $f(x)$ is a continuous function with $0 \leq x, t \leq T$, where $\mathrm{T}$ is constant. Moreover, generalized Abel's integral equation can be considered the following forms

$$
f(x)=\int_{0}^{x} \frac{u(t)}{(x-t)^{\alpha}} d t
$$

And

$$
u(t)=f(x)+\int_{0}^{x} \frac{u(t)}{(x-t)^{\alpha}} d t
$$

Where $0<\alpha<1, f(x) \in C[0, T], 0 \leq x, t \leq T$ and $\mathrm{T}$ is constant.

The paper it organized the following way: In section 2, we present the fractional integral and derivative operators and some their properties. In section 3, we combined fractional technique, Chebyshev polynomials and the collocation method for solving Abel's integral equation. Some examples are investigated in section 4 . The numerical results show the

\author{
D.Sh.Mohamed \\ Department of \\ Mathematics \\ Faculty of Science, \\ University of Zagazig
}

\author{
A.E.Mahdy \\ Department of \\ Mathematics
}

Faculty of Science,

University of Zagazig

accuracy of the method. Last section is conclusion that gives some points of the method.

\section{FRACTIONAL INTEGRAL AND DERIVATIVE}

Definition 2.1. A real function $u(x), x>0$, is said to be in the space $C_{\mu}, \mu \in R$ if there exists a real number $(p>$ $\mu) \square \square$ such that $u(x)=x^{p} v(x)$, where $v(x) C[0, \infty), \square$ and it is said to be in the space $C_{\mu}^{m}$, iff $u^{(m)} \in C_{\mu}, m \in N$.

Definition 2.2. The Riemann-Liouville fractional integral operator of order $\alpha \geq 0$, of a function $u(x) \in C_{\mu}, \mu \geq-1$ is define as

$$
J^{\alpha} u(x)=\frac{1}{\Gamma(\alpha)} \int_{0}^{x}(x-t)^{\alpha-1} u(t) d t
$$

such that $J^{0} u(x)=u(x)$

proposition 2.1. The operator $J^{\alpha}$ in definition 2.2 satisfies the following properties for $u_{i} \in C_{\mu}, i=0 \ldots n$, $\mu \geq-1$.

1. $J^{\alpha}\left(\sum_{i=0}^{n} u_{i}(x)\right)=\sum_{i=0}^{n} J^{\alpha} u_{i}(x)$,

2. $J^{\alpha} x^{\beta}=\frac{\Gamma(\beta+1)}{\Gamma(\alpha+\beta+1)} x^{\alpha+\beta}, \beta>-1$

Definition 2.3. The Riemann-Liouville fractional derivative operator of order $\alpha \geq 0$, of a function $u(x)$ is define as

$$
\begin{gathered}
D^{\alpha} u(x)=\frac{1}{\Gamma(1-\alpha)} D \int_{0}^{x}(x-t)^{-\alpha} u(t) d t, \\
0<\alpha<1, \quad x>0
\end{gathered}
$$

where $D u \square$ means the first order derivative of $u$.

Definition 2.4. The Caputo fractional derivative of order $\alpha$ is define as

$$
\begin{aligned}
D^{\alpha} f(x) & \\
& =J^{m-\alpha} D^{m} f(x) \\
& =\frac{1}{\Gamma(m-\alpha)} D \int_{0}^{x}(x-t)^{m-\alpha-1} f^{(m)}(t) d t,
\end{aligned}
$$

Where, $\mathrm{m}-1 \leq \alpha \leq m, m \in N, x>0$ it has the following two basic properties

and

$$
D^{\alpha} J^{\alpha} f(x)=f(x)
$$

$$
J^{\alpha} D^{\alpha} f(x)=f(x)-\sum f^{(k)}\left(0^{+}\right) \frac{x^{k}}{k !}, x>0
$$




\section{DESCRIPTION OF THE METHOD}

In this method, we use the Chebyshev polynomials through the fractional calculus to approximate the solution of Abel.s integral equations. So, we introduce briefly orthogonal Chebyshev polynomials as a suitable tool for approximation $[7,18]$.

Definition 3.1. The Chebyshev polynomial $T_{n}(t)$ of the first kind for $t=\cos (\theta),(0 \leq \theta \leq \pi), \square$ the function

$$
T_{n}(t)=\cos (n \theta)=\cos \left(n \cos ^{-1}(t)\right)
$$

is a polynomial of degree $n, T_{n}(t)$ is called Chebyshev polynomial of degree $n$. When $\theta$ increase from 0 to $\pi, \mathrm{t}$ decrease from 1 to -1 . Then the interval $[-1,1]$ is domain of definition of $\operatorname{Tn}(t)$ Also, the roots of Chebyshev polynomial of degree $n+1$ can be obtained by the following formula

$$
t_{i}=\cos \frac{(2 i-1) \pi}{2(n+1)}, \quad i=1, \ldots, n+1
$$

In addition, the successive Chebyshev polynomials can be obtain by the following recursive relation

$$
\begin{aligned}
& T_{0}(x)=1, \\
& T_{1}(x)=x \\
& T_{2}(x)=2 x^{2}-1, \\
& T_{n}(x)=2 x T_{n-1}(x)-T_{n-2}(x), \quad n=2,3,4, \ldots
\end{aligned}
$$

Definition 3.2. The Chebyshev polynomial $U_{n}(t)$ of the second kind for $t=\cos (\theta),(0 \leq \theta \leq \pi)$ the function

$$
U_{n}(t)=\frac{\sin (n+1) \theta}{\sin (\theta)}
$$

is a polynomial of degree $n, U_{n}(t)$ is called Chebyshev polynomial of degree $n$. When $\theta$ increase from 0 to $\pi, \mathrm{t}$ decrease from 1 to -1 . Then the interval $[-1,1]$ is domain of definition of $U_{n}(t)$ Also, the roots of Chebyshev polynomial of degree $n+1$ can be obtained by the following formula

$$
t_{i}=\cos \frac{(2 i-1) \pi}{2(n+2)}, \quad i=1, \ldots, n+1
$$

In addition, the successive Chebyshev polynomials can be obtain by the following recursive relation

$$
\begin{aligned}
& U_{0}(x)=1, \\
& U_{1}(x)=2 x \\
& U_{2}(x)=4 x^{2}-1, \\
& U_{n}(x)=2 x U_{n-1}(x)-U_{n-2}(x), \quad n=2,3,4, \ldots
\end{aligned}
$$

Definition 3.3. The Chebyshev polynomial $V_{n}(t)$ of the second kind for $t=\cos (\theta),(0 \leq \theta \leq \pi)$ the function

$$
V_{n}(t)=\frac{\cos \left(n+\frac{1}{2}\right) \theta}{\cos \frac{\theta}{2}}
$$

is a polynomial of degree $n, V_{n}(t)$ is called Chebyshev polynomial of degree $n$. When $\theta$ increase from 0 to $\pi, \mathrm{t}$ decrease from 1 to -1 . Then the interval $[-1,1]$ is domain of definition of $\left.V_{n}(t)\right)$ Also, the roots of Chebyshev polynomial of degree $n+1$ can be obtained by the following formula

$$
t_{i}=\cos \frac{(2 i) \pi}{(2 n+3)}, \quad i=1, \ldots, n+1
$$

In addition, the successive Chebyshev polynomials can be obtain by the following recursive relation

$$
\begin{aligned}
& V_{0}(x)=1, \\
& V_{1}(x)=2 x-1, \\
& V_{2}(x)=4 x^{2}-2 x-1, \\
& V_{n}(x)=2 x V_{n-1}(x)-V_{n-2}(x), \quad n=2,3,4, \ldots
\end{aligned}
$$

Definition 3.4. The Chebyshev polynomial $W_{n}(t)$ of the second kind for $t=\cos (\theta),(0 \leq \theta \leq \pi)$ the function

$$
W_{n}(t)=\frac{\sin \left(n+\frac{1}{2}\right) \theta}{\sin \left(\frac{1}{2}\right) \theta}
$$

is a polynomial of degree $n, W_{n}(t)$ is called Chebyshev polynomial of degree $n$. When $\theta$ increase from 0 to $\pi, \mathrm{t}$ decrease from 1 to -1 . Then the interval $[-1,1]$ is domain of definition of $W_{n}(t)$ Also, the roots of Chebyshev polynomial of degree $n+1$ can be obtained by the following formula

$$
t_{i}=\cos \frac{(2 i-1) \pi}{(2 n+3)}, \quad i=1, \ldots, n+1
$$

In addition, the successive Chebyshev polynomials can be obtain by the following recursive relation

$$
\begin{aligned}
& W_{0}(x)=1 \\
& W_{1}(x)=2 x+1 \\
& W_{2}(x)=4 x^{2}+2 x-1 \\
& W_{n}(x)=2 x W_{n-1}(x)-W_{n-2}(x), n=2,3,4, \ldots
\end{aligned}
$$

Now, we apply chebyshev polynomials for solving Abel's integral equation of the first and second kind.

\subsection{First kind}

According to (1.3) and (2.1), Abel's integral equation of the first kind can be rewritten as follow

$$
f(x)=\Gamma(1-\alpha) J^{1-\alpha} u(x)
$$

Since calculating of $J^{1-\alpha} u(x)$ is directly cost and inefficient, we will use Chebyshev polynomials for approximating $u(x)$. We assume $u(x)$ on interval $[-1,1]$, can be written as a infinite series of Chebyshev basis

$$
u(x)=\sum_{i=0}^{\infty} a_{i} G_{i}(x)
$$

where $G_{i}(x)=T_{i}(x), U_{i}(x), V_{i}(x)$ and $W_{i}(x)$ are the first, second, third and forth kind of chebyshev polynomials. For interval $[a, b]$, we can use suitable change of variable to obtain this interval. So we express $u(x)$ as a truncated Chebyshev series as follow 


$$
u_{n}(x)=\sum_{i=0}^{n} a_{i} G_{i}(x)
$$

such that $u_{n}(x)$ will be approximated solution of Abel's integral equation. Now, we can write $(3,13)$ in the form

$$
f(x)=\Gamma(1-\alpha) \sum_{i=0}^{n} a_{i} J^{1-\alpha} G_{i}(x)
$$

Note that, we applied the linear combination property of fractional integral according to proposition 2.1. So, it is sufficient to obtain $J^{1-\alpha} G_{i}(x)$. Assume

$$
G_{i}(x)=\sum_{k=0}^{i} b_{k} x^{k}
$$

where $b_{k}, k=0 \ldots i$ are coefficients of Chebyshev polynomial of degree $i$ that are defined by (3.3). Now, by replacing (3.17) in $J^{1-\alpha} G_{i}(x)$ we have

$$
J^{1-\alpha} G_{i}(x)=\sum_{k=0}^{i} b_{k} J^{1-\alpha} x^{k}
$$

Proposition 2.1. confirms validity of (3.17) and utilizes computation of $J^{1-\alpha} T_{i}$. So, substitution (3.18) in (3.16) gives the following form

$$
f(x)=\Gamma(1-\alpha) \sum_{i=0}^{n} a_{i} \sum_{k=0}^{i} b_{k} J^{1-\alpha} x^{k}
$$

Now, we collocate the roots of Chebyshev polynomial of degree $n+1$ in (3.19). It leads to a system of linear equations. By solution of obtained system we have the approximate solution of Abel's integral equation as (3.15). For more efficiency of this method, we suggest reordering Chebychev series as follows

$$
\sum_{i=0}^{n} a_{i} G_{i}(x)=\sum_{i=0}^{n} c_{i} x^{i}
$$

where $c_{i}$ is linear combination of $a_{i}$. Then (3.16) is reformed

$$
f(x)=\Gamma(1-\alpha) \sum_{i=0}^{n} c_{i} J^{1-\alpha} x^{i}
$$

This reformation leads to reduce computation the term $J^{1-\alpha} x^{i}$. We remind using directly $\left\{1, x, \ldots, x^{n}\right\}$ as basis instead of Chebyshev.

\subsection{Second kind}

We can rewrite (1.4) with consideration (2.1) in the form

$$
u(x)=f(x)+\Gamma(1-\alpha) J^{1-\alpha} u(x)
$$

Similarly, we replace (3.15) to (3.22). So we have

$$
\sum_{i=0}^{n} a_{i} G_{i}(x)=f(x)+\Gamma(1-\alpha) \sum_{i=0}^{n} a_{i} J^{1-\alpha} G_{i}(x)
$$

or equivalently by using (3.20)

$$
\sum_{i=0}^{n} a_{i} G_{i}(x)=f(x)+\Gamma(1-\alpha) \sum_{i=0}^{n} c_{i} J^{1-\alpha} x^{i}
$$

After computing $J^{1-\alpha} x^{i}$ and substitute the collocation points we have asystem of linear equations. Solution of the system leads to the approximated solution of Abel's integral equation. We solve some examples by this method and assess the accuracy of method in the next section.

\section{NUMERICAL EXAMPLES}

This section is devoted to computational results. We apply the presented method in this paper and solve several examples. Those examples are chosen whose exact solutions exist.All of the computations have been done using the Maple 16.

Example.1. Consider Abel's integral equation

$$
\int_{0}^{x} \frac{u(t)}{\sqrt{(x-t)}} d t=\frac{2}{105} \sqrt{x}\left(105-56 x^{2}+48 x^{3}\right)
$$

with the exact solution $x^{3}-x^{2}+1[17,3,21]$. Applying chebyshev polynomials of the first kind to integral equation (4.1) at $n=3$, we obtain the approximate solution which is the same as the exact solution. Similarly in all caces of chebyshev polynomials

Note : We note that if the exact solution is a polynomial of degree $\mathrm{k}$, the solution is the same as the exact solution for all $n>k$.

Example.2. Consider the following Abel's integral equation of the second kind

$$
u(x)=x^{2}+\frac{16}{15} x^{\frac{5}{2}}-\int_{0}^{x} \frac{u(t)}{\sqrt{(x-t)}} d t
$$

with exact solution $x^{2}[15,17,3,21]$. Similar to the previous example, Applying chebyshev polynomials of the second kind to integral equation (4.2) at $n=2$, we obtain the approximate solution which is the same as the exact solution. Similarly in all cases of chebyshev polynomials.

Example.3. Consider the following Abel's integral equation of the second kind

$$
u(x)=\frac{1}{2} \pi x+\sqrt{x}-\int_{0}^{x} \frac{u(t)}{\sqrt{(x-t)}} d t
$$

with exact solution $u(x)=\sqrt{x} \quad$ [3]. The numerical results are shown in table 1 .

Example.4. Consider the following Abel's integral equation of the first kind 


$$
\int_{0}^{x} \frac{u(t)}{\sqrt{(x-t)}} d t=\frac{1}{2} \pi x
$$

with exact solution $u(x)=\sqrt{x}$. The numerical results are shown in table 2 .

Example.5. Consider the following Abel's integral equation of the first kind

$$
\int_{0}^{x} \frac{u(t)}{(x-t)^{\frac{1}{3}}} d t=\frac{2}{3 \sqrt{3}} \pi x
$$

with the exact solution $u(x)=x^{\frac{1}{3}}$. The numerical results are shown in table 3 .

Example.6. Consider the following Abel's integral equation of the second kind

$$
u(x)=\frac{1}{2 \sqrt{2}} \pi x+x^{\frac{1}{4}}-\int_{0}^{x} \frac{u(t)}{(x-t)^{\frac{1}{4}}} d t
$$

with exact solution $u(x)=x^{\frac{1}{4}}$. The numerical results are shown in table 4.

Table 1: Estimate the exact , approximate solution and error of Example 3.

\begin{tabular}{|c|c|c|c|c|c|c|c|c|c|}
\hline \multicolumn{2}{|c|}{$\begin{array}{c}\text { Chebyshev at } \\
n=5\end{array}$} & \multicolumn{2}{|c|}{ First kind } & \multicolumn{2}{|c|}{ Second kind } & \multicolumn{2}{|c|}{ Third kind } & \multicolumn{2}{|c|}{ Fourth kind } \\
\hline$x$ & Exact & Approximate & Abs.Error & Approximate & Abs.Error & Approximate & Abs.Error & Approximate & Abs.Error \\
\hline 0.1 & 0.3162277660 & 0.3096574554 & 0.0065703106 & 0.09650710208 & 0.2197206639 & 0.1081840013 & 0.2080437647 & 0.07941293031 & 0.2368148357 \\
\hline 0.2 & 0.4472135955 & 0.4383222520 & 0.0088913435 & 0.1837835895 & 0.2634300060 & 0.2009414857 & 0.2462721098 & 0.1782092498 & 0.2690043457 \\
\hline 0.3 & 0.5477225575 & 0.5641447309 & 0.0164221734 & 0.2627601481 & 0.2849624094 & 0.2799339030 & 0.2677886545 & 0.2674991351 & 0.2802234224 \\
\hline 0.4 & 0.6324555320 & 0.6634064973 & 0.0309509653 & 0.3336195724 & 0.2988359596 & 0.3436918146 & 0.2887637174 & 0.3444498621 & 0.2880056699 \\
\hline 0.5 & 0.7071067812 & 0.7316987094 & 0.0245919282 & 0.3963925876 & 0.3107141936 & 0.3940059987 & 0.3131007825 & 0.4080523267 & 0.2990544545 \\
\hline 0.6 & 0.7745966692 & 0.7761659683 & 0.0015692991 & 0.4517554768 & 0.3228411924 & 0.4355618482 & 0.3390348210 & 0.4592908605 & 0.3153058087 \\
\hline 0.7 & 0.8366600265 & 0.8108917730 & 0.0257682535 & 0.5014894547 & 0.3351705718 & 0.4764192978 & 0.3602407287 & 0.5016469736 & 0.3350130529 \\
\hline 0.8 & 0.8944271910 & 0.8531739009 & 0.0412532901 & 0.5482220071 & 0.3462051839 & 0.5290043915 & 0.3654227995 & 0.5413950124 & 0.3530321786 \\
\hline 0.9 & 0.9486832981 & 0.9319331428 & 0.0167501553 & 0.5946638006 & 0.3540194975 & 0.6116873125 & 0.3369959856 & 0.5877273581 & 0.3609559400 \\
\hline 1.0 & 1.0 & 1.124303414 & 0.124303414 & 0.6438172447 & 0.3561827553 & 0.75020747 & 0.2497739253 & 0.6533237187 & 0.3466762813 \\
\hline
\end{tabular}

\begin{tabular}{|c|c|c|c|c|c|c|c|c|c|}
\hline \multicolumn{2}{|c|}{$\begin{array}{c}\text { Chebyshev at } \\
n=5\end{array}$} & \multicolumn{2}{c|}{ First kind } & \multicolumn{2}{c|}{ Second kind } & \multicolumn{2}{c|}{ Third kind } & \multicolumn{2}{c|}{ Fourth kind } \\
\hline$x$ & Exact & Approximate & Abs.Error & Approximate & Abs.Error & Approximate & Abs.Error & Approximate & Abs.Error \\
\hline 0.1 & 0.3162277660 & 0.3262289843 & 0.0100012183 & 0.1633385109 & 0.1528892551 & 0.3118947197 & 0.0043330463 & 0.2860710870 & 0.0301566790 \\
\hline 0.2 & 0.4472135955 & 0.4370911793 & 0.0101224162 & 0.3382916170 & 0.1089219785 & 0.4284104918 & 0.0188031037 & 0.4118411422 & 0.0353724533 \\
\hline 0.3 & 0.5477225575 & 0.5514814746 & 0.0037589171 & 0.5116141282 & 0.0361084293 & 0.5371406971 & 0.0105818604 & 0.5445463708 & 0.0031761867 \\
\hline 0.4 & 0.6324555320 & 0.6504046356 & 0.0179491036 & 0.6639959271 & 0.0315403951 & 0.6303329948 & 0.0021225372 & 0.6594473042 & 0.0269917722 \\
\hline 0.5 & 0.7071067812 & 0.7275839923 & 0.0204772111 & 0.7782388816 & 0.0711321004 & 0.7088529798 & 0.0017461986 & 0.7467007601 & 0.0395939789 \\
\hline 0.6 & 0.7745966692 & 0.7843527148 & 0.0097560456 & 0.8460402700 & 0.0714436008 & 0.7759456911 & 0.0013490219 & 0.8050278933 & 0.0304312241 \\
\hline 0.7 & 0.8366600265 & 0.8277730839 & 0.0088869426 & 0.8734083956 & 0.0367483691 & 0.8342817618 & 0.0023782647 & 0.8412925127 & 0.0046324862 \\
\hline 0.8 & 0.8944271910 & 0.8695680595 & 0.0248591315 & 0.8841209990 & 0.0103061920 & 0.8883016507 & 0.0061255403 & 0.8714991594 & 0.0229280316 \\
\hline 0.9 & 0.9486832981 & 0.9273276526 & 0.0213556455 & 0.9207990287 & 0.0278842694 & 0.9589703168 & 0.0102870187 & 0.9224970442 & 0.261862539 \\
\hline 1.0 & 1.0 & 1.032310535 & 0.032310535 & 1.049560627 & 0.049560627 & 1.115689009 & 0.115689009 & 1.034768546 & 0.034768546 \\
\hline
\end{tabular}

Table 2 : Estimate the exact, approximate solution and error of Example 4.

Table 3 : Estimate the exact, approximate solution and error of Example 5.

\begin{tabular}{|c|c|c|c|c|c|c|c|c|c|}
\hline \multicolumn{2}{|c|}{$\begin{array}{c}\text { Chebyshev at } \\
n=5\end{array}$} & \multicolumn{2}{c|}{ First kind } & \multicolumn{2}{c|}{ Second kind } & \multicolumn{2}{c|}{ Third kind } & \multicolumn{2}{c|}{ Fourth kind } \\
\hline$x$ & Exact & Approximate & Abs.Error & Approximate & Abs.Error & Approximate & Abs.Error & Approximate & Abs.Error \\
\hline 0.1 & 0.4641588834 & 0.4569993988 & 0.0071594846 & 0.5007413075 & 0.0365824241 & 0.4219664362 & 0.0421924472 & 0.4178182891 & 0.0463405943 \\
\hline 0.2 & 0.5848035476 & 0.5648062624 & 0.0199972852 & 0.5647654482 & 0.0200380994 & 0.5304792281 & 0.0543243195 & 0.5581733339 & 0.0266302137 \\
\hline 0.3 & 0.6694329501 & 0.6741151721 & 0.0046822220 & 0.6446275115 & 0.0248054386 & 0.6359209305 & 0.0335120196 & 0.7045568396 & 0.0351238895 \\
\hline 0.4 & 0.7368062997 & 0.7567189773 & 0.0199126776 & 0.7212234573 & 0.0155828424 & 0.7347652689 & 0.0020410308 & 0.8113846895 & 0.0745783898 \\
\hline 0.5 & 0.7937005260 & 0.8091090403 & 0.0154085143 & 0.7863475338 & 0.0073529922 & 0.8384039045 & 0.04477033785 & 0.8613622282 & 0.0676617022 \\
\hline 0.6 & 0.8434326653 & 0.8430983615 & 0.0003343038 & 0.8402400594 & 0.0031926059 & 0.9368654900 & 0.0934328247 & 0.8611466043 & 0.0177139390 \\
\hline 0.7 & 0.8879040017 & 0.8727473801 & 0.0151566216 & 0.8866552229 & 0.0012487788 & 0.9820057276 & 0.0941017259 & 0.8393594463 & 0.0485445554 \\
\hline 0.8 & 0.9283177667 & 0.9037438304 & 0.0245739363 & 0.9275154108 & 0.0008023559 & 0.9342787222 & 0.0059609555 & 0.8409844892 & 0.0873332775 \\
\hline 0.9 & 0.9654893846 & 0.9610088165 & 0.0044805681 & 0.9631994230 & 0.0022899616 & 1.034528994 & 0.0690396094 & 0.9363116813 & 0.0291777033 \\
\hline 1.0 & 1 & 1.199736936 & 0.199736936 & 1.011724263 & 0.011724263 & 2.026969975 & 1.026969975 & 1.282205790 & 0.282205790 \\
\hline
\end{tabular}


Table 4 : Estimate the exact , approximate solution and error of Example 6.

\begin{tabular}{|c|c|c|c|c|c|}
\hline \multicolumn{2}{|c|}{$\begin{array}{c}\text { Chebyshev at } \\
n=5\end{array}$} & \multicolumn{2}{c|}{ First kind } & \multicolumn{2}{c|}{ Fourth kind } \\
\hline$x$ & Exact & Approximate & Abs.Error & Approximate & Abs.Error \\
\hline 0.1 & 0.5623413252 & 0.6414337651 & 0.0084209913 & 0.5216812833 & 0.0406600419 \\
\hline 0.2 & 0.6687403050 & 0.6414337651 & 0.0273065399 & 0.6442374879 & 0.0245028171 \\
\hline 0.3 & 0.7400828045 & 0.7333926615 & 0.0066901430 & 0.7732884738 & 0.0332056693 \\
\hline 0.4 & 0.7952707288 & 0.8039736577 & 0.0087029289 & 0.8641481389 & 0.0688774101 \\
\hline 0.5 & 0.8408964153 & 0.8504766728 & 0.0095802575 & 0.8995728907 & 0.0586764754 \\
\hline 0.6 & 0.8801117368 & 0.8845770520 & 0.0044653152 & 0.8887329864 & 0.0086212496 \\
\hline 0.7 & 0.9146912192 & 0.9156105062 & 0.0009192870 & 0.8626871131 & 0.0520041061 \\
\hline 0.8 & 0.9457416090 & 0.9387555460 & 0.0069860630 & 0.8630102041 & 0.0827314049 \\
\hline 0.9 & 0.9740037464 & 0.9740746809 & 0.0000709345 & 0.9570313674 & 0.0169723790 \\
\hline 1.0 & 1 & 1.218950038 & 0.218950038 & 1.336801386 & 0.336801386 \\
\hline
\end{tabular}

We note that the second and third kind of chebyshev polynomials are similar.

\section{CONCLUSION}

In this method, we develop the Chebyshev method through the fractional calculus for solving generalized Abel's integral equations. We note that this method is easy to compute. Also, ability and efficiency of the method are great. In particular, when the exact solution of the problem is polynomial, the method gives the exact solution.

\section{REFERENCES}

[1] A.A. Kilbas, H.M. Srivastava, J.J. Trujillo, Theory and Application of Fractional Differential Equations, NorthHolland Mathematics studies, Vol.204, Elsevier, 2006.

[2] A.D. Polyanin, A.V. Manzhirov, Handbook of Integral Equations, CRC Press, 2008.

[3] Abbas Saadatmandia, Mehdi Dehghanb, A Collocation Method for Solving Abel's Integral Equations of First and Second Kinds,Verlag der Zeitschrift für Naturforschung,752 -- 756 (2008).

[4] C.J. Cremers, R.C. Birkebak, Application of the Abel Integral Equation to Spectrographic Data, Appl. Opt. 5 (1966) 1057-1064.

[5] G. Capobianco, D. Conte, An efficient and fast parallel method for Volterra integral equations of Abel type, J. Comput. Appl. Math. 189 (2006) 481- 493.

[6] I. Podlubny, Fractional Differential Equations, Academic Press, San Diego, CA, 1999.

[7] J.C. Mason, D.C. Handscomb, Chebyshev Polynomials, CRC Press LLC, 2003.

[8] Johin Viley, Sons ,Inc, An introduction to the fractional calculus and fractional differential equations (1993).

[9] K.B. Oldham, J. Spanier, The Fractional Calculus, Academic Press, NewYork, 1974.

[10] K.E. Atkinson, The Numerical Solutions of Integral Equations of the Second Kind, Cambridge University Press, 1997.
[11] K.S. Miller, B. Ross, An Introduction to the Fractional Calculus and Fractional Differential Equations,Wiley, NewYork, 1993.

[12] L. Huang, Y. Huang, X.F. Li, Approximate solution of Abel integral equation, Comput. Math. Appl. 56 (2008) 1748-1757.

[13] M. Rahman, Integral Equations and Their Application, WITpress, 2007.

[14] R. Estrada, R.P. Kanwal, Singular Integral Equations, Springer, 2000

[15] R. Gorenflo, S. Vessella, Abel Integral Equations: Analysis and Applications. Lecture Notes in Mathematics 1461, Springer-Verlag, Berlin, 1991.

[16] R.K. Pandey, O.P. Singh, V.K. Singh, Efficient algorithms to solve singular integral equations of Abel type, 57 (2009) 664-676.

[17] R.P. Agarwal, D. O'Regan, Singular Differential and Integral Equations with Applications, Springer, 2003.

[18] S.A. Yousefi, Numerical solution of Abel,s integral equation by using Legendre wavelets, Appl. Math. Comput. 175 (2006) 574-580.

[19] samah M. Dardery, Mohamed M. Allan, Chebyshev Polynomials for Solving a Class of Singular Integral Equations, Applied Mathematics, 2014, 5, 753-764.

[20] T. Miyakoda, Discretized fractional calculus with a series of Chebyshev polynomial, Electron. Notes Theor Comput. Sci. 225 (2009) 239-244.

[21] V. Mirceski, Z. Tomovski, Analytical solutions of integral equations for modelling of reversible electrode processes under voltammetric conditions, J. Electroanal. Chem. 619-620 (2008) 164-168.

[22] Z. Avazzadeh, B. Shafiee and G. B. Loghmani, Fractional Calculus for Solving Abel's Integral Equations Using Chebyshev Polynomials, Applied Mathematical Sciences, Vol. 5, 2011, no. 45, 2207 - 2216. 